\title{
Impact of body mass index on surgical outcomes, narcotics consumption, and hospital costs following anterior cervical discectomy and fusion
}

\author{
Ankur S. Narain, BA, Fady Y. Hijji, MD, Brittany E. Haws, BS, Krishna T. Kudaravalli, BS, \\ Kelly H. Yom, BA, Jonathan Markowitz, BS, and Kern Singh, MD \\ Department of Orthopaedic Surgery, Rush University Medical Center, Chicago, Illinois
}

\begin{abstract}
OBJECTIVE Given the increasing prevalence of obesity, more patients with a high body mass index (BMI) will require surgical treatment for degenerative spinal disease. In previous investigations of lumbar spine pathology, obesity has been associated with worsened postoperative outcomes and increased costs. However, few studies have examined the association between BMI and postoperative outcomes following anterior cervical discectomy and fusion (ACDF) procedures. Thus, the purpose of this study was to compare surgical outcomes, postoperative narcotics consumption, complications, and hospital costs among BMI stratifications for patients who have undergone primary 1- to 2-level ACDF procedures.
\end{abstract}

METHODS The authors retrospectively reviewed a prospectively maintained surgical database of patients who had undergone primary 1- to 2-level ACDF for degenerative spinal pathology between 2008 and 2015. Patients were stratified by BMI as follows: normal weight $\left(<25.0 \mathrm{~kg} / \mathrm{m}^{2}\right)$, overweight $\left(25.0-29.9 \mathrm{~kg} / \mathrm{m}^{2}\right)$, obese I $\left(30.0-34.9 \mathrm{~kg} / \mathrm{m}^{2}\right)$, or obese II-III $\left(\geq 35.0 \mathrm{~kg} / \mathrm{m}^{2}\right)$. Differences in patient demographics and preoperative characteristics were compared across the BMI cohorts using 1-way ANOVA or chi-square analysis. Multivariate linear or Poisson regression with robust error variance was used to determine the presence of an association between BMI category and narcotics utilization, improvement in visual analog scale (VAS) scores, incidence of complications, arthrodesis rates, reoperation rates, and hospital costs. Regression analyses were controlled for preoperative demographic and procedural characteristics.

RESULTS Two hundred seventy-seven patients were included in the analysis, of whom $20.9 \%(n=58)$ were normal weight, $37.5 \%(n=104)$ were overweight, $24.9 \%(n=69)$ were obese I, and $16.6 \%(n=46)$ were obese II-III. A higher BMI was associated with an older age $(p=0.049)$ and increased comorbidity burden $(p=0.001)$. No differences in sex, smoking status, insurance type, diagnosis, presence of neuropathy, or preoperative VAS pain scores were found among the BMI cohorts $(p>0.05)$. No significant differences were found among these cohorts as regards operative time, intraoperative blood loss, length of hospital stay, and number of operative levels $(p>0.05)$. Additionally, no significant differences in postoperative narcotics consumption, VAS score improvement, complication rates, arthrodesis rates, reoperation rates, or total direct costs existed across BMI stratifications ( $p>0.05$ ).

CONCLUSIONS Patients with a higher BMI demonstrated surgical outcomes, narcotics consumption, and hospital costs comparable to those of patients with a lower BMI. Thus, ACDF procedures are both safe and effective for all patients across the entire BMI spectrum. Patients should be counseled to expect similar rates of postoperative complications and eventual clinical improvement regardless of their BMI.

https://thejns.org/doi/abs/10.3171/2017.6.SPINE17288

KEY WORDS body mass index; ACDF; narcotics; outcomes; complications; direct cost; cervical

$\mathrm{O}$ BESITY is a health epidemic in the United States, with $35.5 \%$ of the adult population considered to be obese according to body mass index (BMI). ${ }^{26}$ Excess weight and obesity have been linked to significant comorbidities including diabetes, cardiovascular disease, and obstructive sleep apnea. ${ }^{1,15,18}$ A high BMI can also have adverse effects on spinal health, as evidenced by the increased rates of spinal degenerative disease among obese patients. ${ }^{13}$ Regarding the cervical spine, obese patients have an increased risk of cervical myelopathy and

ABBREVIATIONS ACDF = anterior cervical discectomy and fusion; $\mathrm{BMI}=$ body mass index; $\mathrm{CCl}=\mathrm{Charlson}$ Comorbidity Index; $\mathrm{POD}=$ postoperative day; $\mathrm{VAS}=$ visual analog scale.

SUBMITTED March 7, 2017. ACCEPTED June 20, 2017.

INCLUDE WHEN CITING Published online December 1, 2017; DOI: 10.3171/2017.6.SPINE17288. 
radiculopathy resulting from intervertebral disc degeneration and chronic back pain..$^{12,22}$ When conservative methods fail to adequately treat these debilitating conditions, surgery is often used to provide symptom relief and improve the patient's quality of life.

Anterior cervical discectomy and fusion (ACDF) is a safe and effective surgical intervention that is commonly used to treat degenerative cervical spine pathology. ${ }^{10,21}$ However, both physicians and hospital systems have expressed concern about the impact of obesity on the outcomes and costs of spinal procedures. Previous studies have demonstrated that obesity is associated with longer operative times and increased blood loss during spinal surgery. ${ }^{5,8}$ Additionally, previous investigations have revealed that obese patients undergoing spinal fusion incur $27 \%$ higher costs than nonobese patients, with ACDF specifically reported to have lower cost-effectiveness in morbidly obese patients. ${ }^{6,9}$ Furthermore, concomitant chronic obstructive pulmonary disease and obesity have been correlated with a longer hospital stay and increased hospital charges in women who have undergone ACDF. ${ }^{17}$ However, prior investigations have documented no differences in patient-reported outcomes between obese and nonobese patients who have undergone ACDF. ${ }^{19}$ While the impact of obesity on outcomes is a common topic in the literature, few studies have specifically analyzed the effect of obesity on a comprehensive set of outcomes including inpatient pain and narcotics use, hospital costs, and postoperative pain for patients who have undergone ACDF procedures.

Improved knowledge of the association between BMI and surgical outcomes may allow for improved patient selection and counseling. Furthermore, investigations into the association between BMI and surgical costs of ACDF may lead to reduced health care expenditures. Therefore, the purpose of this study was to determine if there is an association between BMI and surgical outcomes, postoperative narcotics consumption, complications, and costs for patients who underwent ACDF procedures.

\section{Methods \\ Patient Population}

After obtaining institutional review board approval, we retrospectively reviewed our institution's prospectively maintained surgical registry to identify patients who had undergone primary 1- to 2-level ACDF between 2008 and 2015. Surgical indications included central and foraminal stenosis, degenerative disc disease, and herniated nucleus pulposus. All patients underwent ACDF performed by a single surgeon at our academic institution. Patients were excluded from this study if 2 years had not elapsed since their index procedure or if they had undergone surgery for traumatic etiologies. The study population consisted of consecutive patients with the exception of those removed because of exclusion criteria.

\section{Surgical Technique}

The Smith-Robinson approach was used for all procedures, and implanted interbody cages were packed with autograft, allograft, or bone graft substitute. ${ }^{20}$ Supplemental plate fixation was used as necessary.

\section{Demographics, Comorbidity, and Clinical Outcomes Analysis}

Patients were grouped by BMI category as follows: normal weight $\left(<25.0 \mathrm{~kg} / \mathrm{m}^{2}\right)$, overweight $(25.0-29.9 \mathrm{~kg}$ / $\left.\mathrm{m}^{2}\right)$, obese I $\left(30.0-34.9 \mathrm{~kg} / \mathrm{m}^{2}\right)$, and obese II-III $(\geq 35.0$ $\mathrm{kg} / \mathrm{m}^{2}$ ). Patient demographic and comorbidity information was obtained from the prospectively maintained surgical registry. Of note, the comorbidity burden was expressed as a modified form of the Charlson Comorbidity Index (CCI) with the age component removed. This allowed for the use of both age and CCI as independent variables in the multivariate regression analyses. Neurological examinations had been performed on all patients as part of the preoperative medical evaluation, and all findings were documented. Operative information included the number of operative levels, operative time, intraoperative estimated blood loss, and duration of inpatient stay. Postoperative inpatient narcotics consumption was measured and converted to units of oral morphine equivalents using the conversion methodology described by Gordon et al. ${ }^{7}$ Narcotics consumption was expressed as both the total and hourly amounts consumed on postoperative day (POD) 0 and POD 1.

Patients completed the visual analog scale (VAS) questionnaire to assess their perceived level of neck pain at the preoperative, immediate postoperative, and postoperative follow-up time points ( 6 weeks, 12 weeks, 6 months). The VAS rates pain on a scale of 0 (no pain) to 10 (extreme pain). ${ }^{25}$ Other measured outcomes included the inpatient and postoperative complication rates, arthrodesis rates, and reoperation rates. Arthrodesis was defined as the presence of bony bridging on 3 sequential coronal and sagittal sections on CT scans routinely performed at 6 months after surgery. If fusion was not achieved at 6 months postoperatively and the patient was symptomatic at 1 year postoperatively, an additional CT scan was performed. The incidence of reoperation was evaluated for a 2 -year period after each index procedure.

\section{Cost Analyses}

Direct costs were obtained from our institution's administrative records. Direct costs consisted of hospital charges for blood products, cardiology, emergency room, radiology/imaging, laboratory, nursing unit, intensive care unit, pharmacy, physical/occupational/speech therapy, surgical services, and other. If patients required a revision arthrodesis procedure at the index level, the costs associated with both the primary and the revision surgical encounter were aggregated to determine the total cost of operative therapy for the affected level. Total costs were tabulated and represent the sum of direct and overhead costs.

\section{Statistical Analysis}

Statistical analysis was performed using Stata/MP 13.1 for Mac (StataCorp LP). Body mass index category was tested for an association with demographic, comorbidity, and operative characteristics using chi-square analysis or 1-way ANOVA for categorical and continuous variables, respectively. Multivariate linear or Poisson regression with robust error variance was used to determine the 
TABLE 1. Baseline characteristics of 277 patients who underwent ACDF

\begin{tabular}{|c|c|c|c|c|c|}
\hline Parameter & Normal Weight & Overweight & Obese I & Obese II-III & p Value* \\
\hline No. of patients & 58 & 104 & 69 & 46 & \\
\hline Mean age in yrs & $49.5 \pm 11.3$ & $48.8 \pm 10.2$ & $51.8 \pm 9.2$ & $53.4 \pm 11.2$ & 0.049 \\
\hline Sex & & & & & 0.272 \\
\hline $\mathrm{F}$ & $29(50)$ & $44(42)$ & $25(36)$ & $24(52)$ & \\
\hline M & $29(50)$ & $60(58)$ & $44(64)$ & $22(48)$ & \\
\hline Smoking status & & & & & 0.172 \\
\hline Smoker & $43(74)$ & $77(74)$ & $59(86)$ & $39(85)$ & \\
\hline Nonsmoker & $15(26)$ & $27(26)$ & $10(14)$ & $7(15)$ & \\
\hline Insurance type & & & & & 0.765 \\
\hline Non-WC & $38(66)$ & $67(64)$ & $43(62)$ & $33(72)$ & \\
\hline WC & $20(34)$ & $37(36)$ & $26(38)$ & $13(28)$ & \\
\hline \multicolumn{6}{|l|}{ Diagnosis $†$} \\
\hline Herniated nucleus pulposus & $53(91)$ & $90(87)$ & $55(80)$ & $36(78)$ & 0.172 \\
\hline Degenerative disc disease & $0(0)$ & $9(9)$ & $5(7)$ & $1(2)$ & 0.077 \\
\hline Central stenosis & $8(14)$ & $31(30)$ & $20(29)$ & $16(35)$ & 0.069 \\
\hline Foraminal stenosis & $3(5)$ & $1(1)$ & $4(6)$ & $1(2)$ & 0.261 \\
\hline Neuropathy & & & & & 0.113 \\
\hline Radiculopathy & $29(50)$ & $32(31)$ & $22(32)$ & $12(26)$ & \\
\hline Myelopathy & $0(0)$ & $2(2)$ & $1(1)$ & $2(4)$ & \\
\hline Myeloradiculopathy & $27(47)$ & $60(58)$ & $40(58)$ & $31(67)$ & \\
\hline Diabetes status & & & & & $<0.001$ \\
\hline No diabetes mellitus & $57(98)$ & $98(94)$ & $60(87)$ & $27(59)$ & \\
\hline Diabetes mellitus & $1(2)$ & $6(6)$ & $9(13)$ & $19(41)$ & \\
\hline Mean modified CCl & $1.5 \pm 0.9$ & $1.7 \pm 1.2$ & $1.9 \pm 1.2$ & $2.4 \pm 1.2$ & 0.001 \\
\hline Mean preop VAS neck score & $6.7 \pm 2.0$ & $6.2 \pm 2.0$ & $6.3 \pm 2.0$ & $6.6 \pm 1.8$ & 0.521 \\
\hline
\end{tabular}

presence of an association between BMI category and narcotics utilization, improvement in VAS pain scores, incidence of complications, arthrodesis rates, reoperation rates, and costs. Multivariate analyses were adjusted for patient age, sex, smoking status, diabetes status, modified CCI, and number of operative levels. Statistical significance was set at $\mathrm{p}<0.05$. Patients with missing data points were excluded from statistical analysis for that particular variable.

\section{Power Analysis}

Power analysis revealed the present study has 91\% power to detect a difference of $\$ 1500$ in average total costs between normal/overweight groups (162 patients) and obese I-III groups (115 patients).

\section{Results}

\section{Patient Demographics and Operative Characteristics}

A total of 302 patients underwent primary 1- to 2-level ACDF in the study period. Twenty-five patients $(8.3 \%)$ were lost to follow-up; thus, 277 patients were included in this analysis. Among the study patients, $20.9 \%$ (58 pa- tients) were normal weight, $37.5 \%(\mathrm{n}=104)$ were overweight, $24.9 \%(n=69)$ were obese I, and $16.6 \%(n=46)$ were obese II-III. Table 1 presents data regarding baseline demographic and comorbidity characteristics in the study population. The mean ages of the patients in the higher BMI categories were older than those in the lower categories (normal weight: 49.5 years; overweight: 48.8; obese I: 51.8; and obese II-III: 53.4; $\mathrm{p}=0.049)$. A higher BMI category was also associated with a greater comorbidity burden as measured by modified CCI (normal weight: 1.5 ; overweight: 1.7; obese I: 1.9; obese II-III: $2.4 ; \mathrm{p}=0.001$ ). Furthermore, patients with a higher BMI were more likely to be diabetic than those with a lower BMI (normal weight: 2\%; overweight: 6\%; obese I: $13 \%$; obese II-III: $41 \%$; $<$ $0.001)$. There were no statistically significant differences among the BMI groups with regard to sex, smoking status, insurance type, diagnosis, presence of neuropathy, or preoperative VAS neck pain score ( $p>0.05$ for each).

Table 2 presents information regarding operative characteristics. The BMI category was not associated with significant differences in operative time, intraoperative estimated blood loss, duration of inpatient stay, or number of operative levels ( $\mathrm{p}>0.05$ for each). 
TABLE 2. Perioperative outcomes in patients who underwent ADCF

\begin{tabular}{lccccc}
\hline \multicolumn{1}{c}{ Parameter } & Normal Weight & Overweight & Obese I & Obese II-III & p Value* \\
\hline No. of patients & 58 & 104 & 69 & 46 & \\
\hline Mean operative time in mins & $61.1 \pm 20.4$ & $56.2 \pm 17.5$ & $63.2 \pm 22.9$ & $62.8 \pm 21.2$ & 0.107 \\
\hline Mean estimated blood loss in $\mathrm{ml}$ & $46.6 \pm 16.5$ & $47.9 \pm 28.2$ & $51.2 \pm 30.6$ & $45.8 \pm 15.1$ & 0.641 \\
\hline Mean length of hospital stay in hrs & $31.9 \pm 22.5$ & $31.5 \pm 16.6$ & $37.2 \pm 22.9$ & $36.7 \pm 18.0$ & 0.170 \\
\hline No. of operative levels & & & & & 0.109 \\
\hline 1 & $29(50)$ & $66(63)$ & $38(55)$ & $20(43)$ & \\
\hline 2 & $29(50)$ & $38(37)$ & $31(45)$ & $26(57)$ & \\
\hline
\end{tabular}

Values expressed as the mean \pm standard deviation or as number of patients (\%).

* Calculated by chi-square analysis or 1-way ANOVA.

Table 3 presents data regarding inpatient VAS pain scores and narcotics consumption on POD 0 and POD 1. There were no significant differences in postoperative pain among BMI groups on POD 0 or POD 1 ( $p>0.05$ for each). Additionally, BMI category was not associated with significant differences in daily or hourly narcotics consumption on either POD 0 or POD 1 ( $p>0.05$ for each).

Table 4 presents data regarding postoperative outcomes. There were no differences among the BMI groups in terms of VAS neck pain score improvement at the 6-week, 12-week, or 6-month postoperative time point ( $p>0.05$ for each). The complication, arthrodesis, and reoperation rates were also similar among the different BMI cohorts ( $p>0.05$ for each). Complications included urinary retention requiring catheterization (24 cases), reintubation ( $\mathrm{n}=$ $2)$, epidural hematoma $(\mathrm{n}=1)$, altered mental status $(\mathrm{n}=$ $1)$, and instrumentation failure $(n=4)$. Indications for reoperation included pseudarthrosis (9 cases), adjacent-segment degeneration $(\mathrm{n}=13)$, residual stenosis $(\mathrm{n}=3)$, and ossification of the posterior longitudinal ligament $(n=2)$.

\section{Cost Analyses}

Cost analyses are presented in Table 5. There were no significant differences in total costs among the BMI cohorts $(p=0.965)$. Upon categorization of total costs, BMI category was associated with average "other" costs (normal weight: $\$ 53$; overweight: $\$ 52$; obese I: $\$ 61$; obese IIIII: $\$ 132 ; p=0.028)$. There were no significant differences among BMI cohorts for any other individual cost category ( $p>0.05$ for each).

\section{Discussion}

Obesity is an increasingly prevalent public health concern for both patients and medical practitioners. Multiple studies have demonstrated an association between obesity and adverse outcomes following lumbar surgery. ${ }^{5,8,9}$ However, there is a paucity of literature regarding obesity's influence on anterior cervical surgery. Thus, our purpose in this study was to determine if there is an association between preoperative BMI and outcomes, narcotics utilization, and hospital costs after ACDF procedures.

Results of this study indicated that there are no differences among BMI groups as regards outcomes of ACDF, including pain improvement, complication rate, arthrodesis rate, and reoperation rate. Additionally, there were no differences in immediate postoperative pain or inpatient narcotics consumption among BMI groups. Furthermore, total direct hospital costs were similar regardless of a patient's BMI status. These findings suggest that patients with a higher BMI are not worse surgical candidates for

TABLE 3. Inpatient pain scores and narcotics consumption

\begin{tabular}{lcccc}
\hline \multicolumn{1}{c}{ Parameter } & Normal Weight & Overweight & Obese I & Obese II-III $^{p \text { Value }}$ \\
\hline No. of patients & 58 & 104 & 69 & 46 \\
\hline Mean VAS pain scores & & & & \\
\hline POD 0 & $4.9 \pm 1.8$ & $5.0 \pm 1.7$ & $4.7 \pm 1.9$ & $4.9 \pm 1.8$ \\
\hline POD 1 & $3.9 \pm 1.8$ & $4.3 \pm 1.7$ & $3.7 \pm 2.0$ & $4.1 \pm 1.5$ \\
\hline Mean daily OME consumption & & & & \\
\hline POD 0 & $55.8 \pm 46.7$ & $67.7 \pm 58.4$ & $73.5 \pm 65.5$ & $58.0 \pm 51.5$ \\
\hline POD 1 & $33.6 \pm 20.9$ & $38.1 \pm 35.7$ & $44.8 \pm 38.7$ & $42.0 \pm 35.0$ \\
\hline Mean hourly OME consumption & & & & 0.254 \\
\hline POD 0 & $4.5 \pm 3.5$ & $6.5 \pm 11.9$ & $6.1 \pm 6.0$ & $4.8 \pm 4.9$ \\
\hline POD 1 & $2.1 \pm 1.3$ & $2.3 \pm 1.9$ & $2.5 \pm 1.8$ & $2.4 \pm 2.0$ \\
\hline
\end{tabular}

OME = oral morphine equivalent.

Values expressed as the mean \pm standard deviation.

* Calculated using multivariate linear regression controlled for age, sex, smoking status, diabetes status, modified CCl, and number of fusion levels. 
TABLE 4. Postoperative outcomes in patients who underwent ACDF

\begin{tabular}{|c|c|c|c|c|c|}
\hline Parameter & Normal Weight & Overweight & Obese I & Obese II-III & p Value* \\
\hline No. of patients & 58 & 104 & 69 & 46 & \\
\hline \multicolumn{6}{|c|}{ Mean change in VAS neck score $\dagger$} \\
\hline 6 wks & $-3.1 \pm 2.5(56)$ & $-3.4 \pm 2.4(101)$ & $-2.6 \pm 3.1(65)$ & $-2.9 \pm 2.4(44)$ & 0.233 \\
\hline 12 wks & $-3.9 \pm 3.1(55)$ & $-3.8 \pm 2.7(98)$ & $-3.8 \pm 3.2(64)$ & $-3.2 \pm 3.0(43)$ & 0.391 \\
\hline $6 \mathrm{mos}$ & $-4.2 \pm 3.2(54)$ & $-4.2 \pm 2.9(100)$ & $-4.3 \pm 3.4(63)$ & $-3.9 \pm 2.3(42)$ & 0.794 \\
\hline Complications $\ddagger$ & $8(14)$ & $14(13)$ & $4(6)$ & $4(9)$ & 0.258 \\
\hline Pseudarthrosis & $3(5)$ & $6(6)$ & $3(4)$ & $2(4)$ & - \\
\hline Arthrodesis at $1 \mathrm{yr}$ & $55(95)$ & $98(94)$ & $66(96)$ & $44(96)$ & 0.940 \\
\hline Reoperations§ & $5(9)$ & $11(11)$ & $8(12)$ & $3(7)$ & 0.968 \\
\hline
\end{tabular}

- = not applicable.

Values expressed as the mean \pm standard deviation or as number of patients (\%).

* From multivariate Poisson or linear regression adjusted for age, sex, smoking status, diabetes status, modified CCI, and number of operative levels.

$\dagger$ Numbers in parentheses represent the number of patients with data for that particular data point analysis.

$\ddagger$ Complications included urinary retention requiring catheterization $(24$ cases), reintubation $(n=2)$, epidural hematoma $(n=1)$, altered mental status $(n=1)$, and instrumentation failure $(n=4)$.

$\S$ Indications for reoperation included pseudarthrosis ( 9 cases), adjacent-segment degeneration $(n=13)$, residual stenosis $(n=3)$, and ossification of the posterior longitudinal ligament $(n=2)$.

ACDF procedures and that they should be counseled to expect short-term and long-term outcomes similar to those of patients with a lower BMI.

While results of the current study indicated that postoperative pain improvement is similar among BMI groups, the literature has exhibited mixed results. In a study of 299 patients who underwent ACDF, Sielatycki et al. determined that obese and nonobese patients had similar improvements in numeric rating scale (NRS) pain scores up to 1 year postoperatively. ${ }^{19}$ Conversely, Auffinger et al. demonstrated that obese patients undergoing anterior or posterior cervical procedures had increased VAS scores at 6 months postoperatively compared with the scores of nonobese patients. ${ }^{3}$ However, these authors' inclusion of both anterior and posterior cervical procedures among the study population hinders their ability to make definitive conclusions regarding the effect of BMI on ACDF procedures alone.

In the current study we also determined that a higher BMI was not associated with increased intraoperative or postoperative complications. This result is similar to that of Buerba et al., who performed a study of 4071 patients undergoing cervical fusion procedures. ${ }^{4}$ These authors stratified patients into nonobese $\left(18.5-29.9 \mathrm{~kg} / \mathrm{m}^{2}\right)$, obese I $\left(30.0-34.9 \mathrm{~kg} / \mathrm{m}^{2}\right)$, obese II $\left(35.0-39.9 \mathrm{~kg} / \mathrm{m}^{2}\right)$, and obese III $\left(\geq 40.0 \mathrm{~kg} / \mathrm{m}^{2}\right)$ groups. In the patients who underwent anterior cervical fusion, a high BMI was not associated with increased complication rates during the first 30 postoperative days. But conflicting findings exist in the literature, with Kalanithi et al. demonstrating that morbidly

TABLE 5. Direct costs for patients who underwent ACDF

\begin{tabular}{|c|c|c|c|c|c|}
\hline Parameter & Normal Weight & Overweight & Obese I & Obese II-III & $p$ Value ${ }^{*}$ \\
\hline No. of patients & 58 & 104 & 69 & 46 & \\
\hline Mean total costs & $\$ 9097 \pm 4264$ & $\$ 8605 \pm 3345$ & $\$ 8839 \pm 2816$ & $\$ 9006 \pm 3112$ & 0.965 \\
\hline Blood & $\$ 0 \pm 0$ & $\$ 2 \pm 10$ & $\$ 2 \pm 9$ & $\$ 1 \pm 8$ & 0.171 \\
\hline Cardiology & $\$ 10 \pm 68$ & $\$ 15 \pm 118$ & $\$ 5 \pm 26$ & $\$ 22 \pm 83$ & 0.696 \\
\hline ER & $\$ 0 \pm 0$ & $\$ 2 \pm 22$ & $\$ 0 \pm 0$ & $\$ 0 \pm 0$ & 0.514 \\
\hline Radiology & $\$ 169 \pm 65$ & $\$ 157 \pm 41$ & $\$ 171 \pm 56$ & $\$ 159 \pm 46$ & 0.249 \\
\hline Laboratory & $\$ 26 \pm 57$ & $\$ 20 \pm 25$ & $\$ 24 \pm 29$ & $\$ 23 \pm 27$ & 0.436 \\
\hline Nursing unit & $\$ 731 \pm 611$ & $\$ 789 \pm 573$ & $\$ 919 \pm 901$ & $\$ 823 \pm 536$ & 0.241 \\
\hline ICU & $\$ 101 \pm 603$ & $\$ 0 \pm 0$ & $\$ 22 \pm 183$ & $\$ 25 \pm 171$ & 0.168 \\
\hline Pharmacy & $\$ 345 \pm 224$ & $\$ 331 \pm 132$ & $\$ 356 \pm 195$ & $\$ 357 \pm 155$ & 0.776 \\
\hline PT/OT/speech therapist & $\$ 82 \pm 74$ & $\$ 85 \pm 68$ & $\$ 96 \pm 85$ & $\$ 94 \pm 62$ & 0.873 \\
\hline Surgical services & $\$ 7579 \pm 3384$ & $\$ 7153 \pm 2855$ & $\$ 7181 \pm 2014$ & $\$ 7369 \pm 2805$ & 0.977 \\
\hline Other & $\$ 53 \pm 124$ & $\$ 52 \pm 113$ & $\$ 61 \pm 130$ & $\$ 132 \pm 245$ & 0.028 \\
\hline
\end{tabular}

$\mathrm{ER}=$ emergency room; $\mathrm{PT}=$ physical therapy; $\mathrm{OT}=$ occupational therapy.

Values expressed as the mean \pm standard deviation. Boldface type indicates statistical significance.

* From multivariate linear regression adjusted for age, sex, smoking status, diabetes status, modified $\mathrm{CCl}$, and number of operative levels. 
obese patients $\left(\geq 40 \mathrm{~kg} / \mathrm{m}^{2}\right)$ exhibited higher complication rates than normal-weight patients after undergoing anterior cervical fusion procedures. ${ }^{9}$ The most common complications in these morbidly obese patients were wound and pulmonary in nature. However, it is important to note that the investigation by Kalanithi et al. included only morbidly obese and normal-weight patients and thus does not represent the pattern of complication incidence over the totality of the BMI spectrum.

Rates of arthrodesis and the need for reoperation were also similar across the BMI spectrum in the present study. This suggests that patients, regardless of their BMI status, should be counseled similarly regarding the risks of undergoing an operation subsequent to their index procedure. This finding is in agreement with much of the literature regarding both cervical and lumbar fusion procedures. ${ }^{8,14,23}$ In a study of 672 patients undergoing ACDF, van Eck et al. determined that the need for revision was not affected by variables such as BMI, age, sex, smoking status, or number of levels fused..$^{23}$ Owens et al., utilizing propensity-matched cohorts among 560 lumbar fusion patients with long-term follow-up, demonstrated that the revision rate and time between the index and revision surgeries did not differ among normal-weight, overweight, and obese groups. ${ }^{14}$ However, Jiang et al. presented contrasting findings in a meta-analysis of patients undergoing spine surgery, noting that obese patients had higher revision rates than nonobese patients. ${ }^{8}$ It is important to note, however, that the majority of revisions in that study were attributable to recurrent disc herniations after decompression procedures without fusion. This may indicate that BMI and body habitus play a different biomechanical role following decompression-only spinal procedures compared with fusion procedures.

Narcotics utilization and pain in the immediate postoperative period is a significant concern after spinal procedures such as ACDF. This concern is magnified in obese patients, who typically have increased comorbidities and a higher risk of obstructive sleep apnea. ${ }^{1,15,18}$ Thus, these patients often require close monitoring of analgesic intake postoperatively to avoid opioid-induced respiratory depression and other opioid-related adverse events. ${ }^{15}$ However, it is imperative to balance the risk of opioid-induced adverse events with adequate analgesia to ensure that patients do not experience complications such as atelectasis and subsequent pneumonia due to pain. ${ }^{1}$ The current study demonstrated that patients across the BMI spectrum experience similar levels of postoperative pain and consume similar amounts of narcotic analgesics during their postoperative inpatient stay. A similar result has been demonstrated by Armaghani et al., who determined that BMI was not a risk factor for increased opioid demand in the postanesthesia care unit (PACU) in patients who had undergone elective spinal surgery. ${ }^{2}$ These findings suggest that patients with a higher BMI are probably not at risk for excessive opioid intake, and thus postoperative analgesic regimens after ACDF should not be altered based on a patient's BMI. However, narcotics administration will remain an aspect of care that requires constant reevaluation, especially with the advent of new dosing strategies and the increased prevalence of multimodal analgesia protocols.
Another important factor in determining the utility and economic viability of ACDF procedures is the direct hospital costs. Drivers of increased costs can include patient comorbidities, the incidence of postoperative complications, and the length of the hospital stay., ${ }^{9}, 11,16$ The present study demonstrated that total hospital costs were similar among all included BMI groups. This result is not unexpected as our cohort demonstrated no differences in operative time, complication rate, or length of stay according to BMI status. However, these results differ from much of the published literature. ${ }^{6,9,11,16,17,24}$ In a study of 1082 patients undergoing ACDF, Minhas et al. determined that obesity was associated with a \$681 increase in direct hospital costs. ${ }^{11}$ Interestingly, these authors found no association between obesity and common cost drivers such as increased hospital stay, complications, or operative time. They postulated that the increased costs may have stemmed from complications requiring more expensive treatment or an increased utilization of surgical supplies in obese patients. Similarly, Puvanesarajah et al. determined that obesity was associated with a $\$ 651$ increase in Medicare reimbursements in older patients undergoing 1- to 2-level ACDF. ${ }^{16}$ The previously mentioned study by Kalanithi et al. also noted that morbidly obese patients incurred higher complication rates and higher direct costs than the normal-weight patients. ${ }^{9}$ Because that study only included morbidly obese or normal-weight patients, however, it is unclear whether valid comparisons can be made to studies including patients over more inclusive BMI ranges. Nonetheless, the scope of the literature supports focusing cost-reduction efforts toward complication avoidance and length-of-stay reduction.

This study is not without limitations. First, it was performed with a cohort of patients treated by a single surgeon at a single site. Thus, the generalizability of the data may be limited. Second, we did not separately analyze patients with a BMI $>40 \mathrm{~kg} / \mathrm{m}^{2}$ or patients who had undergone 3- to 4-level procedures since the number of patients in these cohorts was low. This may limit the accuracy of our results in these specific subsets of patients. Third, the retrospective nature of this study may be associated with selection bias. Fourth, information on preoperative narcotics use was unavailable. Thus, we could not determine the effect of tolerance on both inpatient pain and narcotics utilization postoperatively. Fifth, postdischarge narcotics use was not tracked; therefore, we could not quantify the effect of continued analgesic use on postoperative pain scores at the follow-up time points. Sixth, data on additional patientreported outcome measures such as the Neck Disability Index or SF-12 Physical and Mental Composite scores were unavailable for much of this patient population because of survey noncompliance. Similarly, poor compliance with patient-reported outcome surveys at the 1- and 2-year postoperative time points limited our long-term analysis. Thus, patient-reported outcome data were presented up to 6 months postoperatively since compliance was highest at that point. Finally, long-term cost utility could not be determined from hospital charges alone. Further investigation into the long-term cost-effectiveness of ACDF procedures is required to fully elucidate the durability and efficacy of anterior cervical surgery. 


\section{Conclusions}

As the prevalence of obesity increases, so will the need for surgical treatment of cervical spine pathology in patients with a higher BMI. The most common of these treatments will be ACDF procedures. Therefore, understanding the relationship between BMI and outcomes of ACDF is imperative for patient selection and risk stratification. In this study, patients spanning the entire BMI spectrum exhibited similar postoperative outcomes, narcotics utilization rates, and total direct costs after 1- to 2-level ACDF procedures. Given these results, surgeons should not preclude patients from undergoing $\mathrm{ACDF}$ procedures based solely on BMI or body habitus. Additionally, practitioners should not use BMI as a basis for altering postoperative analgesia protocols. Finally, similar postoperative outcomes should be expected regardless of a patient's preoperative BMI status.

\section{References}

1. Alvarez A, Singh PM, Sinha AC: Postoperative analgesia in morbid obesity. Obes Surg 24:652-659, 2014

2. Armaghani SJ, Lee DS, Bible JE, Archer KR, Shau DN, Kay $\mathrm{H}$, et al: Preoperative opioid use and its association with perioperative opioid demand and postoperative opioid independence in patients undergoing spine surgery. Spine (Phila Pa 1976) 39:E1524-E1530, 2014

3. Auffinger B, Lam S, Kraninger J, Shen J, Roitberg BZ: The impact of obesity on surgeon ratings and patient-reported outcome measures after degenerative cervical spine disease surgery. World Neurosurg 82:e345-e352, 2014

4. Buerba RA, Fu MC, Grauer JN: Anterior and posterior cervical fusion in patients with high body mass index are not associated with greater complications. Spine J 14:1643-1653, 2014

5. Cao J, Kong L, Meng F, Zhang Y, Shen Y: Impact of obesity on lumbar spinal surgery outcomes. J Clin Neurosci 28:1-6, 2016

6. Chotai S, Sielatycki JA, Parker SL, Sivaganesan A, Kay HL, Stonko DP, et al: Effect of obesity on cost per qualityadjusted life years gained following anterior cervical discectomy and fusion in elective degenerative pathology. Spine J 16:1342-1350, 2016

7. Gordon DB, Stevenson KK, Griffie J, Muchka S, Rapp C, Ford-Roberts K: Opioid equianalgesic calculations. J Palliat Med 2:209-218, 1999

8. Jiang J, Teng Y, Fan Z, Khan S, Xia Y: Does obesity affect the surgical outcome and complication rates of spinal surgery? A meta-analysis. Clin Orthop Relat Res 472:968-975, 2014

9. Kalanithi PA, Arrigo R, Boakye M: Morbid obesity increases cost and complication rates in spinal arthrodesis. Spine (Phila Pa 1976) 37:982-988, 2012

10. McClelland S III, Oren JH, Protopsaltis TS, Passias PG: Outpatient anterior cervical discectomy and fusion: a metaanalysis. J Clin Neurosci 34:166-168, 2016

11. Minhas SV, Chow I, Jenkins TJ, Dhingra B, Patel AA: Preoperative predictors of increased hospital costs in elective anterior cervical fusions: a single-institution analysis of 1,082 patients. Spine J 15:841-848, 2015

12. Nilsen TI, Holtermann A, Mork PJ: Physical exercise, body mass index, and risk of chronic pain in the low back and neck/shoulders: longitudinal data from the Nord-Trondelag Health Study. Am J Epidemiol 174:267-273, 2011

13. Nouri A, Tetreault L, Singh A, Karadimas SK, Fehlings MG: Degenerative cervical myelopathy: epidemiology, genetics, and pathogenesis. Spine (Phila Pa 1976) 40:E675-E693, 2015
14. Owens RK II, Djurasovic M, Onyekwelu I, Bratcher KR, McGraw KE, Carreon LY: Outcomes and revision rates in normal, overweight, and obese patients 5 years after lumbar fusion. Spine J 16:1178-1183, 2016

15. Porhomayon J, Leissner KB, El-Solh AA, Nader ND: Strategies in postoperative analgesia in the obese obstructive sleep apnea patient. Clin J Pain 29:998-1005, 2013

16. Puvanesarajah V, Kirby DJ, Jain A, Werner BC, Hassanzadeh $\mathrm{H}$ : Cost variation of anterior cervical fusions in elderly Medicare beneficiaries. Spine (Phila Pa 1976) 42:E1010-E1015, 2017

17. Sami Walid M, Zaytseva NV: The impact of chronic obstructive pulmonary disease and obesity on length of stay and cost of spine surgery. Indian J Orthop 44:424-427, 2010

18. Schug SA, Raymann A: Postoperative pain management of the obese patient. Best Pract Res Clin Anaesthesiol 25:7381,2011

19. Sielatycki JA, Chotai S, Kay H, Stonko D, McGirt M, Devin CJ: Does obesity correlate with worse patient-reported outcomes following elective anterior cervical discectomy and fusion? Neurosurgery 79:69-74, 2016

20. Smith GW, Robinson RA: The treatment of certain cervicalspine disorders by anterior removal of the intervertebral disc and interbody fusion. J Bone Joint Surg Am 40-A:607-624, 1958

21. Song KJ, Choi BY: Current concepts of anterior cervical discectomy and fusion: a review of literature. Asian Spine J 8:531-539, 2014

22. Teraguchi M, Yoshimura N, Hashizume H, Muraki S, Yamada H, Minamide A, et al: Prevalence and distribution of intervertebral disc degeneration over the entire spine in a population-based cohort: the Wakayama Spine Study. Osteoarthritis Cartilage 22:104-110, 2014

23. van Eck CF, Regan C, Donaldson WF, Kang JD, Lee JY: The revision rate and occurrence of adjacent segment disease after anterior cervical discectomy and fusion: a study of 672 consecutive patients. Spine (Phila Pa 1976) 39:2143-2147, 2014

24. Walid MS, Sanoufa M, Robinson JS: The effect of age and body mass index on cost of spinal surgery. J Clin Neurosci 18:489-493, 2011

25. Williamson A, Hoggart B: Pain: a review of three commonly used pain rating scales. J Clin Nurs 14:798-804, 2005

26. Witek AM, Benzel EC: Obesity in cervical spine surgery. World Neurosurg 82:e147-e148, 2014

\section{Disclosures}

No funds were received in support of this work. No benefits in any form have been or will be received from any commercial party related directly or indirectly to the subject of this paper.

\section{Author Contributions}

Conception and design: Singh, Narain, Hijji. Acquisition of data: Narain, Hijji, Kudaravalli, Yom. Analysis and interpretation of data: Singh, Narain, Hijji, Haws, Markowitz. Drafting the article: Narain, Hijji, Haws. Critically revising the article: Singh, Narain, Hijji, Haws, Markowitz. Reviewed submitted version of manuscript: all authors. Approved the final version of the manuscript on behalf of all authors: Singh. Statistical analysis: Narain, Hijji, Haws. Administrative/technical/material support: Singh, Haws, Kudaravalli, Yom. Study supervision: Singh.

\section{Correspondence}

Kern Singh, Department of Orthopaedic Surgery, Rush University Medical Center, 1611 W Harrison St., Ste. 300, Chicago, IL 60612. email: kern.singh@rushortho.com. 